\title{
Cognitive Profile in Tramadol Addicts
}

By: Saber Hadad Mahdi. Assistant Lecturer of Psychiatry.Faculty of M edicine-Sohag University

Prof.Dr.Hameed M ustafa Baddary. Professor of Psychiatry.Faculty of medicineSohag University

Prof.Dr.MahaWasfiM obasher. Professor of Psychiatry.Faculty of medicine-Cairo University

Dr. TarekDesoky Ahmed. Lecturer of Psychiatry.Faculty of M edicine-Sohag University

\begin{abstract}
Background and Objective: Cognitive impairment is one of the consequencesof substance abuse. Tramadol abuse is a public health problemin Egypt. The objective of this study was to study the effect of tramadol addiction on cognitive functions, determine if cognitive impairment caused by tramadol is reversible or not and to study the impact of other related factors as duration of intake and dose of tramadol on cognition.
\end{abstract}

Methods: This study included 30 patients with active tramadol abuse, 30 patients abstinent from tramadol for 3 to 6 months and 30 control subjects (matched for age, sex, and education) who were recruited from Kasr Al-Aini psychiatric and addiction hospital (from outpatient clinic and inpatient admitted patients), Egypt.The participants were interviewed using Montreal Cognitive Assessmenttest (MoCA), Trail making test $\mathrm{A}$ and $\mathrm{B}$ and had urine screening for drugs.

Results: $53.33 \%(n=16)$ of active group had impaired total MoCA scores compared to $10 \% \quad(\mathrm{n}=3)$ in control group. This was statistically significant with $\mathrm{P}$ value $<0.0001 .46 .67 \% \quad(\mathrm{n}=14)$ of abstinent group had impaired total MoCA scores compared to $10 \%(\mathrm{n}=3)$ in control group. This was statistically significant with $\mathrm{P}$ value 0.002. As regard MoCAsubscores; we found statistically significant impairment in delayed recall and verbal fluency subscores in both the active and abstinent group. There was no statistically significant impairment in Trail making tests A and B.Therewas no association between cognitive impairment and sociodemographicor clinical factors.

Conclusions: Cognitive impairment occurs commonly among tramadolabusepatients. Memory impairment and verbal fluencyare the most common cognitive domainsto be affected. Cognitive impairment is not reversible after 3 to 6 months. There is no effect of tramadol dose or duration of abuse on cognitive impairment.

\section{INTRODUCTION}

Tramadol hydrochloride is a centrallyacting synthetic analogue of codeine; used for the treatment of moderate to severe pain. It has a dual mechanism of action. Tramadol and its active metabolite, O-desmethyl tramadol, bind to $\mu$ opioid receptors, thus exerting their effect on GABAergic transmission. They also inhibit reuptake of 5-hydroxy tryptamine (serotonin) and noradrenaline (Shadnia, et al. 2008, and Taghaddosinejad, et al. 2011).

Few works studied the cognitive profile of tramadol dependence however, many studies were done regarding the cognitive profile of other substances of abuse particularly the 
opioids and cannabis. This can be explained by that tramadol is not a very common drug in the western world and does not represent a medical issue of importance regarding dependence to be a focus for the scientific research unlike in Egypt where it represents a major health issue (Abo-Elmagd, et al. 2013).

\section{SUBJECS AND METHODS}

Subjects

This study compares between three groups regarding cognitive functions. The first group is composed of 30 patients with two years or more of regular tramadol abuse (Dependency). The second group is composed of 30 patients who are abstinent from tramadol intake for three to six months (early recovery stage). The third group is a control group of 30 persons from relatives of the first and second group who are matched with them regarding age, sex and educational level. Convenient sample was recruited from Kasr Al-Aini psychiatric and addiction hospital (from outpatient clinic and inpatient admitted patients).

Inclusion criteria:

1. Patients fulfill criteria for the diagnosis of tramadol dependence based on DSM-5 criteria.

2. Age from 18:50 years.

3. At least 12 years of education.

\section{Exclusion criteria:}

1. Patients with other substance related or addictive disorders.

2. Patients with comorbid psychiatric disorder that may affect cognition.

4. General medical condition that may affect cognition e.g. DM, HTN, Parkinson's disease, Huntington's disease, etc.

\section{Methods}

Each patient in the study was subjected to the following:

A. Psychiatric interview: Using semi structural interview derived from Kasr Al-Aini Sheet with special emphasis on age, occupation, marital status, socioeconomic status and substance history including age of onset, dose, duration, causes of intake and side effects (including any seizures during intake or withdrawal).

2. Physical examination Including temperature, blood pressure, pulse, heart, chest and abdominal examination to exclude general conditions that may affect cognition.

\section{Routine} neurological examination:Standardized neurological examination.

4. Liver and kidney functions and random blood sugar.

5. Urine toxicological screen for Tramadol, Cannabinoids, Benzodiazepines and Opioids to exclude effect of other substances on cognition.

6. Addiction Severity Index Scale (McLellan, et al.1992). Arabic version (Qassem, et al. 2003).

7. To assess cognitive functions:

A.Montreal cognitive assessment (MoCA) (Nasreddine, et al. 2005).Arabic version(Rahman and $E l$ Gaafary, 2009). The Arabic version of MoCA as a prominent tool for evaluation of MCI. The MoCA test is a one-page 30-point test administered in approximately 10 minutes. Each participant had the test individually. It was used to assess several domains of cognition such as the short-term memory recall task (5 points) involves two learning trials of five nouns and delayed recall after approximately 5 minutes. Visuospatial abilities are assessed using a clock-drawing task (3 points) and a three-dimensional cube copy (1 point). Multiple aspects of executive functions are assessed using an alternation task adapted from the trail-making $\mathrm{B}$ task (1 point), a phonemic fluency task (1 point), and a two-item verbal abstraction task (2 points). Attention, concentration and working memory are evaluated using a sustained attention task (target 
SOHAG MEDICAL JOURNAL

Vol. 23 No.2 July 2018

detection using tapping; 1 point), a serial subtraction task (3 points), and digits forward and backward (1 point each). Language is assessed using a three-item confrontation naming task with low-familiarity animals (lion, camel, rhinoceros; 3 points), repetition of two syntactically complex sentences (2 points), and the aforementioned fluency task. Finally, orientation to time and place is evaluated (6 points).

Normal MoCA score is above 25, scores of 25 or less are affected.

B.Trail making test (TMT)(Reitan , 1958): Part A requires the individual to draw lines to connect 25 encircled numbers distributed on a page. Part $\mathrm{A}$ tests visual scanning, numeric sequencing, and visuomotor speed. Part B is similar except the person must alternate between numbers and letters and is believed to be more difficult and takes longer to complete.

\section{RESULTS}

\section{Sociodemographic characteristics}

Table (1): Comparison between "active group", "abstinent group" and "control group" according to sociodemographic characteristics

\begin{tabular}{|c|c|c|c|c|c|c|}
\hline Items & Active group & $\begin{array}{l}\text { Abstinent } \\
\text { group }\end{array}$ & Control group & $\begin{array}{l}\text { P1 } \\
\text { value }\end{array}$ & $\begin{array}{l}\text { P2 } \\
\text { value }\end{array}$ & $\begin{array}{l}\text { P3 } \\
\text { value }\end{array}$ \\
\hline $\begin{array}{l}\text { Age/year } \\
\text { Mean } \pm \text { SD } \\
\text { Median (range) }\end{array}$ & $\begin{array}{l}32.33 \pm 7.54 \\
31.5(22-48)\end{array}$ & $\begin{array}{l}30.67 \pm 5.5 \\
29(23-43)\end{array}$ & $\begin{array}{l}30.37 \pm 6.20 \\
28.5(22-45)\end{array}$ & 0.27 & 0.84 & 0.34 \\
\hline $\begin{array}{l}\text { Education } \\
\text { Diplom } \\
\text { Institute } \\
\text { University } \\
\end{array}$ & $\begin{array}{l}22(73.33 \%) \\
3(10.00 \%) \\
5(16.67 \%) \\
\end{array}$ & $\begin{array}{l}17(56.67 \%) \\
5(16.67 \%) \\
8(26.67 \%) \\
\end{array}$ & $\begin{array}{l}14(46.67 \%) \\
5(16.67 \%) \\
11(36.67 \%) \\
\end{array}$ & 0.10 & 0.68 & 0.40 \\
\hline $\begin{array}{l}\text { Occupation } \\
\text { Not working } \\
\text { Non-skillful } \\
\text { Skillful } \\
\text { Professional } \\
\end{array}$ & $\begin{array}{l}5(16.67 \%) \\
9(30.00 \%) \\
14(46.67 \%) \\
2(6.67 \%) \\
\end{array}$ & $\begin{array}{l}5(16.67 \%) \\
10(33.33 \%) \\
13(43.33 \%) \\
2(6.67 \%) \\
\end{array}$ & $\begin{array}{l}4(13.33 \%) \\
11(36.67 \%) \\
13(43.33 \%) \\
2(6.67 \%) \\
\end{array}$ & 0.95 & 0.98 & 0.99 \\
\hline $\begin{array}{l}\text { Marital status } \\
\text { Single } \\
\text { Married }\end{array}$ & $\begin{array}{l}12(40.00 \%) \\
18(60.00 \%)\end{array}$ & $\begin{array}{l}16(53.33 \%) \\
14(46.67 \%)\end{array}$ & $\begin{array}{l}17(56.67 \%) \\
13(43.33 \%)\end{array}$ & 0.20 & 0.78 & 0.30 \\
\hline $\begin{array}{l}\text { Residence } \\
\text { Rural } \\
\text { Urban }\end{array}$ & $\begin{array}{l}17(56.67 \%) \\
13(43.33 \%)\end{array}$ & $\begin{array}{l}16(53.33 \%) \\
14(46.67 \%)\end{array}$ & $\begin{array}{l}19(63.33 \%) \\
11(36.67 \%)\end{array}$ & 0.60 & 0.43 & 0.80 \\
\hline
\end{tabular}

Table (1) shows no statistically significant difference in age, education, occupation, marital status or residence between the three groups. 
SOHAG MEDICAL JOURNAL

Vol. 23 No.2 July 2018
Cognitive Profile in Tramadol Addicts Saber Hadad Mahdi

N.B. P1 value between "active group" and "control group".P2 value between "abstinent group" and "control group". P3 value between "active group" and

"abstinent group".

\section{Clinical characteristics}

Table (2): Nicotine use in "active group", "abstinent group" and "control group"

\begin{tabular}{|l|l|l|l|l|l|l|}
\hline Item & Active group & Abstinent group & Control group & $\begin{array}{l}\text { P1 } \\
\text { value }\end{array}$ & P2 value & $\begin{array}{l}\text { P3 } \\
\text { value }\end{array}$ \\
\hline $\begin{array}{l}\text { Nicotine use } \\
\text { No } \\
\text { Yes }\end{array}$ & $\begin{array}{l}\mathbf{4}(13.33 \%) \\
\mathbf{2 6}(86.67 \%)\end{array}$ & $\begin{array}{l}\mathbf{7 ( 2 3 . 3 3 \% )} \\
\mathbf{2 3}(\mathbf{7 6 . 6 7 \%})\end{array}$ & $\begin{array}{l}\mathbf{2 1}(\mathbf{7 0 . 0 0 \% )} \\
\mathbf{9 ( 3 0 . 0 0 \% )}\end{array}$ & $\begin{array}{l}<\mathbf{0 . 0 0 0} \\
\mathbf{1}\end{array}$ & $<\mathbf{0 . 0 0 0 1}$ & $\mathbf{0 . 3 2}$ \\
\hline
\end{tabular}

N.B. P1 value between "active group" and "control group".P2 value between "abstinent group" and "control group". P3 value between "active group" and "abstinent group".

Table (3): Seizures in "active group", "abstinent group" and "control group"

\begin{tabular}{|l|l|l|l|l|l|l|}
\hline Item & Active group & Control group & Abstinent group & P1 value & P2 value & P3 value \\
\hline $\begin{array}{l}\text { seizures } \\
\text { No } \\
\text { Yes }\end{array}$ & $\begin{array}{l}27(90.00 \%) \\
\mathbf{3}(10.00 \%)\end{array}$ & $\begin{array}{l}\mathbf{3 0}(100 \%) \\
\mathbf{0}\end{array}$ & $\begin{array}{l}\mathbf{2 6}(\mathbf{8 6 . 6 7 \%}) \\
\mathbf{4 ( 1 3 . 3 3 \% )}\end{array}$ & $\mathbf{0 . 2 3}$ & $\mathbf{0 . 1 1}$ & $\mathbf{1 . 0 0}$ \\
\hline
\end{tabular}

Table (3) shows no significant difference between the three groups in seizures.

\section{Cognitive tests}

Table (6): Comparison between "active group" and "control group" according to MoCA scores

\begin{tabular}{|c|c|c|c|}
\hline Items & Active group & Control group & P value \\
\hline $\begin{array}{l}\text { Total MoCA } \\
\text { Impaired } \\
\text { Normal }\end{array}$ & $\begin{array}{l}16(53.33 \%) \\
14(46.67 \%)\end{array}$ & $\begin{array}{l}3(10.00 \%) \\
27(90.00 \%)\end{array}$ & $<0.0001$ \\
\hline $\begin{array}{l}\text { Total MoCA } \\
\text { Mean } \pm \text { SD } \\
\text { Median (range) }\end{array}$ & $\begin{array}{l}24.67 \pm 3.19 \\
24(19-30)\end{array}$ & $\begin{array}{l}27.67 \pm 2.26 \\
28(21-30)\end{array}$ & 0.0001 \\
\hline $\begin{array}{l}\text { Trail making } \\
\text { Mean } \pm \text { SD } \\
\text { Median (range) }\end{array}$ & $\begin{array}{l}0.8 \pm 0.41 \\
1(0-1)\end{array}$ & $\begin{array}{l}0.9 \pm 0.31 \\
1(0-1)\end{array}$ & 0.28 \\
\hline $\begin{array}{l}\text { VC cube } \\
\text { Mean } \pm \text { SD } \\
\text { Median (range) }\end{array}$ & $\begin{array}{l}0.8 \pm 0.41 \\
1(0-1) \\
\end{array}$ & $\begin{array}{l}0.87 \pm 0.35 \\
1(0-1)\end{array}$ & 0.49 \\
\hline $\begin{array}{l}\text { VC clock } \\
\text { Mean } \pm \text { SD } \\
\text { Median (range) }\end{array}$ & $\begin{array}{l}2.73 \pm 0.52 \\
3(1-3)\end{array}$ & $\begin{array}{l}2.67 \pm 0.48 \\
3(2-3)\end{array}$ & 0.61 \\
\hline $\begin{array}{l}\text { Naming } \\
\text { Mean } \pm \text { SD } \\
\text { Median (range) }\end{array}$ & $\begin{array}{l}2.97 \pm 0.18 \\
3(2-3)\end{array}$ & $\begin{array}{l}2.93 \pm 0.25 \\
3(2-3)\end{array}$ & 0.56 \\
\hline $\begin{array}{l}\text { Attention } \\
\text { Mean } \pm \text { SD } \\
\text { Median (range) }\end{array}$ & $\begin{array}{l}5.3 \pm 0.88 \\
6(3-6) \\
\end{array}$ & $\begin{array}{l}5.57 \pm 0.50 \\
6(5-6) \\
\end{array}$ & 0.15 \\
\hline $\begin{array}{l}\text { Sentence repetition } \\
\text { Mean } \pm \text { SD } \\
\text { Median (range) }\end{array}$ & $\begin{array}{l}1.9 \pm 0.31 \\
2(1-2)\end{array}$ & $\begin{array}{l}1.9 \pm 0.31 \\
2(1-2) \\
\end{array}$ & 1.00 \\
\hline $\begin{array}{l}\text { Verbal fluency } \\
\text { Mean } \pm \text { SD } \\
\text { Median (range) }\end{array}$ & $\begin{array}{l}0.33 \pm 0.48 \\
0(0-1)\end{array}$ & $\begin{array}{l}0.9 \pm 0.40 \\
1(0-2)\end{array}$ & $<0.0001$ \\
\hline $\begin{array}{l}\text { Abstraction } \\
\text { Mean } \pm \text { SD } \\
\text { Median (range) }\end{array}$ & $\begin{array}{l}1.9 \pm 0.40 \\
2(0-2)\end{array}$ & $\begin{array}{l}1.97 \pm 0.18 \\
2(1-2)\end{array}$ & 0.41 \\
\hline $\begin{array}{l}\text { Delayed recall } \\
\text { Mean } \pm \text { SD } \\
\text { Median (range) }\end{array}$ & $\begin{array}{l}2.46 \pm 1.33 \\
2(1-5)\end{array}$ & $\begin{array}{l}4.47 \pm 1.04 \\
5(1-5)\end{array}$ & $<0.0001$ \\
\hline $\begin{array}{l}\text { Orientation } \\
\text { Mean } \pm \text { SD } \\
\text { Median (range) }\end{array}$ & $\begin{array}{l}5.53 \pm 0.57 \\
6(4-6)\end{array}$ & $\begin{array}{l}5.6 \pm 0.50 \\
6(5-6)\end{array}$ & 0.63 \\
\hline
\end{tabular}

Table (6) shows significant difference between the two groups in total MoCA scores and in subscores of verbal fluency and delayed recall. There was no statistically significant difference in other subscores of MoCA.

Table (7): Comparison between "active group" and "control group" according to Trail making $\mathrm{A}$ and $\mathrm{B}$ scores. 
SOHAG MEDICAL JOURNAL

Vol. 23 No.2 July 2018
Cognitive Profile in Tramadol Addicts Saber Hadad Mahdi

\begin{tabular}{|c|c|c|c|}
\hline Items & Active group & Control group & P value \\
\hline Trail A & $44.43 \pm 13.31$ & $43.23 \pm 12.99$ & 0.56 \\
Mean \pm SD & $45(27-96)$ & $40(27-94)$ & \\
Median (range) & $98.87 \pm 46.39$ & $91.63 \pm 36.82$ & 0.71 \\
Trail B & $83(57-210)$ & $82(57-198)$ & \\
Mean \pm SD & Median (range) & &
\end{tabular}

Table (7) shows no significant difference between the two groups Trail making A and $B$ scores.

Table (8): Comparison between "abstinent group" and "control group" according to MoCA scores.

\begin{tabular}{|c|c|c|c|}
\hline Items & Abstinent group & Control group & P value \\
\hline $\begin{array}{c}\text { Total MoCA } \\
\text { Impaired } \\
\text { Normal }\end{array}$ & $\begin{array}{l}14(46.67 \%) \\
16(53.33 \%)\end{array}$ & $\begin{array}{c}3(10.00 \%) \\
27(90.00 \%)\end{array}$ & 0.002 \\
\hline $\begin{array}{c}\text { Total MoCA } \\
\text { Mean } \pm \text { SD } \\
\text { Median (range) }\end{array}$ & $\begin{array}{l}25.4 \pm 3.08 \\
26(19-30)\end{array}$ & $\begin{array}{c}27.67 \pm 2.26 \\
28(21-30)\end{array}$ & 0.002 \\
\hline $\begin{array}{c}\text { Trail making } \\
\text { Mean } \pm \mathrm{SD} \\
\text { Median (range) }\end{array}$ & $\begin{array}{c}0.8 \pm 0.41 \\
1(0-1)\end{array}$ & $\begin{array}{c}0.9 \pm 0.31 \\
1(0-1)\end{array}$ & 0.28 \\
\hline $\begin{array}{c}\text { VC cube } \\
\text { Mean } \pm \text { SD } \\
\text { Median (range) }\end{array}$ & $\begin{array}{c}0.8 \pm 0.41 \\
1(0-1)\end{array}$ & $\begin{array}{c}0.87 \pm 0.35 \\
1(0-1)\end{array}$ & 0.49 \\
\hline $\begin{array}{c}\text { VC clock } \\
\text { Mean } \pm \text { SD } \\
\text { Median (range) }\end{array}$ & $\begin{array}{c}2.57 \pm 0.56 \\
3(1-3)\end{array}$ & $\begin{array}{c}2.67 \pm 0.48 \\
3(2-3)\end{array}$ & 0.46 \\
\hline $\begin{array}{c}\text { Naming } \\
\text { Mean } \pm \text { SD } \\
\text { Median (range) } \\
\end{array}$ & $\begin{array}{c}2.93 \pm 0.25 \\
3(2-3) \\
\end{array}$ & $\begin{array}{c}2.93 \pm 0.25 \\
3(2-3) \\
\end{array}$ & 1.00 \\
\hline $\begin{array}{c}\text { Attention } \\
\text { Mean } \pm \text { SD } \\
\text { Median (range) }\end{array}$ & $\begin{array}{c}5.47 \pm 0.82 \\
6(3-6)\end{array}$ & $\begin{array}{c}5.57 \pm 0.50 \\
6(5-6)\end{array}$ & 0.57 \\
\hline $\begin{array}{c}\text { Sentence repetition } \\
\text { Mean } \pm \text { SD } \\
\text { Median (range) }\end{array}$ & $\begin{array}{c}1.9 \pm 0.31 \\
2(1-2)\end{array}$ & $\begin{array}{c}1.9 \pm 0.31 \\
2(1-2)\end{array}$ & 1.00 \\
\hline $\begin{array}{l}\text { Verbal fluency } \\
\text { Mean } \pm \text { SD } \\
\text { Median (range) }\end{array}$ & $\begin{array}{c}0.4 \pm 0.50 \\
0(0-1)\end{array}$ & $\begin{array}{c}0.9 \pm 0.40 \\
1(0-2)\end{array}$ & 0.0002 \\
\hline $\begin{array}{c}\text { Abstraction } \\
\text { Mean } \pm \text { SD } \\
\text { Median (range) }\end{array}$ & $\begin{array}{c}1.97 \pm 0.18 \\
2(1-2)\end{array}$ & $\begin{array}{c}1.97 \pm 0.18 \\
2(1-2)\end{array}$ & 1.00 \\
\hline $\begin{array}{c}\text { Delayed recall } \\
\text { Mean } \pm \mathrm{SD} \\
\text { Median (range) }\end{array}$ & $\begin{array}{c}2.9 \pm 1.54 \\
2(1-5)\end{array}$ & $\begin{array}{c}4.47 \pm 1.04 \\
5(1-5)\end{array}$ & 0.0001 \\
\hline $\begin{array}{c}\text { Orientation } \\
\text { Mean } \pm \text { SD } \\
\text { Median (range) }\end{array}$ & $\begin{array}{c}5.63 \pm 0.56 \\
6(4-6)\end{array}$ & $\begin{array}{c}5.6 \pm 0.50 \\
6(5-6)\end{array}$ & 0.81 \\
\hline
\end{tabular}

Table (8) shows significant difference between the two groups in total MoCAscores and in subscores of verbal fluency and delayed recall. There was no statistically significant difference in other subscores of MoCA.

Table (9): Comparison between "abstinent group" and "control group" according to Trail making A and B scores.

\begin{tabular}{|c|c|c|c|}
\hline Items & Abstinent group & Control group & P value \\
\hline Trail A & & & \\
Mean \pm SD & $45.67 \pm 13.90$ & $43.23 \pm 12.99$ & 0.35 \\
Median (range) & $44.5(26-97)$ & $40(27-94)$ & \\
\hline Trail B & $95.00 \pm 41.88$ & $91.63 \pm 36.82$ & 0.89 \\
Mean \pm SD & $80(57-210)$ & $82(57-198)$ & \\
Median (range) &
\end{tabular}

Table (9) shows no difference between the two groups in Trail making A and B scores 
SOHAG MEDICAL JOURNAL

Vol. 23 No.2 July 2018
Cognitive Profile in Tramadol Addicts Saber Hadad Mahdi

Table (10): Comparison between "active group" and "abstinent group" according to MoCA scores.

\begin{tabular}{|c|c|c|c|}
\hline Items & Active group & Abstinent group & P value \\
\hline $\begin{array}{c}\text { Total MoCA } \\
\text { Impaired } \\
\text { Normal }\end{array}$ & $\begin{array}{l}16(53.33 \%) \\
14(46.67 \%)\end{array}$ & $\begin{array}{l}14(46.67 \%) \\
16(53.33 \%)\end{array}$ & 0.61 \\
\hline $\begin{array}{c}\text { Total MoCA } \\
\text { Mean } \pm \text { SD } \\
\text { Median (range) } \\
\end{array}$ & $\begin{array}{l}24.67 \pm 3.19 \\
24(19-30)\end{array}$ & $\begin{array}{l}25.4 \pm 3.08 \\
26(19-30)\end{array}$ & 0.37 \\
\hline $\begin{array}{c}\text { Trail making } \\
\text { Mean } \pm \text { SD } \\
\text { Median (range) }\end{array}$ & $\begin{array}{c}0.8 \pm 0.41 \\
1(0-1)\end{array}$ & $\begin{array}{c}0.8 \pm 0.41 \\
1(0-1)\end{array}$ & 1.00 \\
\hline $\begin{array}{c}\text { VC cube } \\
\text { Mean } \pm \text { SD } \\
\text { Median (range) } \\
\end{array}$ & $\begin{array}{c}0.8 \pm 0.41 \\
1(0-1)\end{array}$ & $\begin{array}{c}0.8 \pm 0.41 \\
1(0-1) \\
\end{array}$ & 1.00 \\
\hline $\begin{array}{c}\text { VC clock } \\
\text { Mean } \pm \text { SD } \\
\text { Median (range) }\end{array}$ & $\begin{array}{c}2.73 \pm 0.52 \\
3(1-3)\end{array}$ & $\begin{array}{c}2.57 \pm 0.56 \\
3(1-3)\end{array}$ & 0.24 \\
\hline $\begin{array}{c}\text { Naming } \\
\text { Mean } \pm \text { SD } \\
\text { Median (range) }\end{array}$ & $\begin{array}{c}2.97 \pm 0.18 \\
3(2-3)\end{array}$ & $\begin{array}{c}2.93 \pm 0.25 \\
3(2-3)\end{array}$ & 0.56 \\
\hline $\begin{array}{c}\text { Attention } \\
\text { Mean } \pm \text { SD } \\
\text { Median (range) }\end{array}$ & $\begin{array}{c}5.3 \pm 0.88 \\
6(3-6)\end{array}$ & $\begin{array}{c}5.47 \pm 0.82 \\
6(3-6)\end{array}$ & 0.45 \\
\hline $\begin{array}{c}\text { Sentence repetition } \\
\text { Mean } \pm \text { SD } \\
\text { Median (range) } \\
\end{array}$ & $\begin{array}{c}1.9 \pm 0.31 \\
2(1-2)\end{array}$ & $\begin{array}{c}1.9 \pm 0.31 \\
2(1-2)\end{array}$ & 1.00 \\
\hline $\begin{array}{c}\text { Verbal fluency } \\
\text { Mean } \pm \text { SD } \\
\text { Median (range) }\end{array}$ & $\begin{array}{c}0.33 \pm 0.48 \\
0(0-1)\end{array}$ & $\begin{array}{c}0.4 \pm 0.50 \\
0(0-1)\end{array}$ & 0.60 \\
\hline $\begin{array}{c}\text { Abstraction } \\
\text { Mean } \pm \text { SD } \\
\text { Median (range) }\end{array}$ & $\begin{array}{c}1.9 \pm 0.40 \\
2(0-2)\end{array}$ & $\begin{array}{c}1.97 \pm 0.18 \\
2(1-2)\end{array}$ & 0.41 \\
\hline $\begin{array}{c}\text { Delayed recall } \\
\text { Mean } \pm \mathrm{SD} \\
\text { Median (range) }\end{array}$ & $\begin{array}{c}2.46 \pm 1.33 \\
2(1-5)\end{array}$ & $\begin{array}{c}2.9 \pm 1.54 \\
2(1-5)\end{array}$ & 0.32 \\
\hline $\begin{array}{c}\text { Orientation } \\
\text { Mean } \pm \text { SD } \\
\text { Median (range) }\end{array}$ & $\begin{array}{c}5.53 \pm 0.57 \\
6(4-6)\end{array}$ & $\begin{array}{c}5.63 \pm 0.56 \\
6(4-6)\end{array}$ & 0.49 \\
\hline
\end{tabular}

Table (10): shows no difference in total score or subscores of MoCA between the two groups.

Table (11): Comparison between "active group" and "abstinent group" according to Trail making A and B scores.

\begin{tabular}{|c|c|c|c|}
\hline Items & Active group & Abstinent group & P value \\
\hline Trail A & & & \\
Mean \pm SD & $44.43 \pm 13.31$ & $45.67 \pm 13.90$ & 0.74 \\
Median (range) & $45(27-96)$ & $44.5(26-97)$ & \\
\hline Trail B & & & \\
Mean \pm SD & $98.87 \pm 46.39$ & $95.00 \pm 41.88$ & 0.86 \\
Median (range) & $83(57-210)$ & $80(57-210)$ & \\
\hline
\end{tabular}

Table (11) shows no difference between the two groups in Trail making A and B scores.

Correlates of cognitive impairment 
SOHAG MEDICAL JOURNAL

Vol. 23 No.2 July 2018
Cognitive Profile in Tramadol Addicts Saber Hadad Mahdi

Table (12): Correlation between cognitive impairment and different items in "active group"

\begin{tabular}{|c|c|c|c|}
\hline Items & $\begin{array}{c}\text { Impaired } \\
N=16\end{array}$ & $\begin{array}{c}\text { Normal } \\
N=14\end{array}$ & $P$ value \\
\hline $\begin{array}{c}\text { Age } \\
\text { Mean } \pm \text { SD } \\
\text { Median (range) }\end{array}$ & $\begin{array}{c}31.06 \pm 8.27 \\
28.5(22-48)\end{array}$ & $\begin{array}{c}33.79 \pm 6.61 \\
33.5(22-44)\end{array}$ & 0.33 \\
\hline $\begin{array}{c}\text { Occupation } \\
\text { Not working } \\
\text { Non-skillful } \\
\text { Skillful } \\
\text { Professional } \\
\end{array}$ & $\begin{array}{c}4(25.00 \%) \\
3(18.75 \%) \\
8(50.00 \%) \\
1(6.25 \%)\end{array}$ & $\begin{array}{c}1(7.14 \%) \\
6(42.86 \%) \\
6(42.86 \%) \\
1(7.14 \%)\end{array}$ & 0.40 \\
\hline $\begin{array}{l}\text { Education } \\
\text { Diplom } \\
\text { Institute } \\
\text { University }\end{array}$ & $\begin{array}{l}10(62.50 \%) \\
3(18.75 \%) \\
3(18.75 \%)\end{array}$ & $\begin{array}{c}12(85.71 \%) \\
0 \\
2(14.29 \%)\end{array}$ & 0.20 \\
\hline $\begin{array}{l}\text { Dose of tramadol } \\
\text { Mean } \pm \text { SD } \\
\text { Median (range) } \\
\end{array}$ & $\begin{array}{l}1301.56 \pm 987.94 \\
1100(450-4250)\end{array}$ & $\begin{array}{c}787.5 \pm 285.35 \\
787.5(450-1350) \\
\end{array}$ & 0.19 \\
\hline $\begin{array}{c}\text { Duration of tramadol use } \\
\text { Mean } \pm \text { SD } \\
\text { Median (range) }\end{array}$ & $\begin{array}{l}6.93 \pm 1.77 \\
5.5(4-11) \\
\end{array}$ & $\begin{array}{l}7.86 \pm 1.83 \\
7.5(5-11) \\
\end{array}$ & 0.17 \\
\hline $\begin{array}{c}\text { Age of onset at tramadol use } \\
\text { Mean } \pm \text { SD } \\
\text { Median (range) }\end{array}$ & $\begin{array}{l}25.31 \pm 8.24 \\
22.5(16-44)\end{array}$ & $\begin{array}{l}26.0 \pm 6.78 \\
24(15-39)\end{array}$ & 0.52 \\
\hline $\begin{array}{l}\text { Fits } \\
\text { No } \\
\text { Yes }\end{array}$ & $\begin{array}{c}13(81.25 \%) \\
3(18.75 \%)\end{array}$ & $\begin{array}{c}14(100 \%) \\
0\end{array}$ & 0.23 \\
\hline $\begin{array}{l}\text { Nicotine use } \\
\text { No } \\
\text { Yes }\end{array}$ & $\begin{array}{l}4(25.00 \%) \\
12(75.00 \%)\end{array}$ & $\begin{array}{c}0 \\
14(100 \%)\end{array}$ & 0.10 \\
\hline
\end{tabular}

Table (12): shows no statistically significant correlation between cognitive impairment and age of the patient, occupation, education, dose of tramadol, duration of tramadol use, seizures or nicotine use.

Table (13): Correlation between cognitive impairment and different items in "abstinent group"

\begin{tabular}{|c|c|c|c|}
\hline Items & $\begin{array}{c}\text { Impaired } \\
N=14\end{array}$ & $\begin{array}{c}\text { Normal } \\
N=16\end{array}$ & $P$ value \\
\hline $\begin{array}{c}\text { Age } \\
\text { Mean } \pm \text { SD } \\
\text { Median (range) }\end{array}$ & $\begin{array}{l}32.07 \pm 6.46 \\
30(24-43)\end{array}$ & $\begin{array}{c}29.44 \pm 4.56 \\
28.5(23-39)\end{array}$ & 0.20 \\
\hline $\begin{array}{c}\text { Occupation } \\
\text { Not working } \\
\text { Non-skillful } \\
\text { Skillful } \\
\text { Professional } \\
\end{array}$ & $\begin{array}{l}2(14.29 \%) \\
6(42.86 \%) \\
6(42.86 \%) \\
0 \\
\end{array}$ & $\begin{array}{l}3(18.75 \%) \\
4(25.00 \%) \\
7(43.75 \%) \\
2(12.50 \%)\end{array}$ & 0.47 \\
\hline $\begin{array}{l}\text { Education } \\
\text { Diplom } \\
\text { Institute } \\
\text { University }\end{array}$ & $\begin{array}{l}6(42.86 \%) \\
5(35.71 \%) \\
3(21.43 \%)\end{array}$ & $\begin{array}{l}3(18.75 \%) \\
5(31.25 \%) \\
8(50.00 \%)\end{array}$ & 0.21 \\
\hline $\begin{array}{l}\text { Dose of tramadol } \\
\text { Mean } \pm \text { SD } \\
\text { Median (range) } \\
\end{array}$ & $\begin{array}{c}984.21 \pm 354.97 \\
900(450-1575)\end{array}$ & $\begin{array}{l}1321.88 \pm 651.46 \\
1125(675-2700) \\
\end{array}$ & 0.12 \\
\hline $\begin{array}{c}\text { Duration of tramadol use } \\
\text { Mean } \pm \text { SD } \\
\text { Median (range) }\end{array}$ & $\begin{array}{c}6.86 \pm 2.03 \\
7(3-10)\end{array}$ & $\begin{array}{c}6.25 \pm 1.52 \\
6.5(3-8)\end{array}$ & 0.36 \\
\hline $\begin{array}{c}\text { Age of onset at tramadol use } \\
\text { Mean } \pm \text { SD } \\
\text { Median (range) }\end{array}$ & $\begin{array}{c}19 \pm 0 \\
19(19-19) \\
\end{array}$ & $\begin{array}{c}19 \pm 0 \\
19(19-19) \\
\end{array}$ & 1.00 \\
\hline $\begin{array}{c}\text { Fits } \\
\text { No } \\
\text { Yes }\end{array}$ & $\begin{array}{c}10(71.43 \%) \\
4(28.57 \%)\end{array}$ & $\begin{array}{c}16(100 \%) \\
0\end{array}$ & 0.4 \\
\hline $\begin{array}{c}\text { Nicotine use } \\
\text { No } \\
\text { Yes } \\
\end{array}$ & $\begin{array}{l}5(35.71 \%) \\
9(64.29 \%) \\
\end{array}$ & $\begin{array}{c}2(12.50 \%) \\
14(87.50 \%)\end{array}$ & 0.20 \\
\hline $\begin{array}{c}\text { Duration of tramadol stoppage } \\
\text { Mean } \pm \text { SD } \\
\text { Median (range) }\end{array}$ & $\begin{array}{c}112.93 \pm 19.30 \\
110(90-150)\end{array}$ & $\begin{array}{c}112.19 \pm 10.80 \\
112.5(95-130)\end{array}$ & 0.54 \\
\hline
\end{tabular}


Table (13) shows no statistically significant relation between cognitive impairment and age of the patient, occupation, education, dose of tramadol, duration of tramadol use, seizures or nicotine use.

\section{Statistical analysis}

Data was analyzed using STATA intercooled version 14.2. Quantitative data was represented as mean, standard deviation, median and range. Data was analyzed using student t-test to compare means of two groups. When the data was not normally distributed Mann-Whitney test was used. Qualitative data was presented as number and percentage and compared using either Chi square test or fisher exact test. Graphs were produced by using Excel or STATA program. $\mathrm{P}$ value was considered significant if it was less than 0.05 .

\section{DISCUSSION}

\section{Sociodemographic data}

Age: The mean age in active group was $32.33 \pm 7.54$ years (Mean \pm SD) with an average range 22-48 years. The mean age in control group was $30.37 \pm 6.20$ years (Mean \pm SD) with an average range $22-45$ years. The mean age in the abstinent group was $30.67 \pm 5.59$ years (Mean \pm SD) with an average range 23-43 years. There was no statistically significant difference in age between the three groups. This indicates cross-matching between the three groups regarding age.

Sex : All the studied patients were males. Absence of female patients in the cases groups can be explained by the social circumstances and stigma related to following up with psychiatric or addiction outpatient clinics in Egypt. We chose males as a control group to match with the cases.

Other sociodemographic factors :There was no difference between the three groups in education, marital status, or occupation.

Regarding cognitive tests:Both active and abstinent group showed significant impairment in total MoCA scores in relation to control group. There was no statistically significant difference between active and abstinent group regarding the total MoCAscores. This can be explained by the long duration of tramadol abuse in both groups (about 7 years) which affects cognitive functions and the short period of abstinence (3-6 months) in the abstinent group which was not sufficient to reverse these cognitive effects of prolonged use of tramadol. Regarding subscores of MoCA, we found statistically significant difference between active group and control group in delayed recall and verbal fluency subscores. We also found significant difference in these two subscores (verbal fluency and delayed recall) between the abstinent and control group.

Regarding Trail-A and Trail-B tests scores, we did not find any significant difference between the active and control group or the abstinent and control group or the active and abstinent group.

There are few studies that assessed the cognitive effects of tramadol and their results were controversial. Some studies found that tramadol caused cognitive impairment, other studies found that tramadol did not impair cognition.

Our results were similar to results of Bassiony and his colleagues(2017). Bassiony and his colleagues used MoCAto evaluate cognitive effects of long term tramadol use. Tramadol abuse patients had significant impairment in all cognitive domains except attention, visuoconstructional (cube), and trailmaking test. The most affected cognitive domains were memory, 
visuospatial skills, and verbal fluency. There was no association between cognitive impairment and dose of tramadol, duration of tramadol abuse, or age at onset of tramadol abuse.

Also, our results were in agreement with the results of Zakaryaee, et al. (2012), who assessed cognitive functions in 26 tramadol dependent patients (who were abstinent for 30-90 days) with 26 non-users control group matched for age, education and male gender. The average dose of tramadol was $900 \mathrm{mg}$ per day (range 400-1300) and average duration of tramadol use was 14.6 month. They found significant impairment in neuropsychological performance in the tramadol use group.

We found two studies that assessed cognitive effects of tramadol used as preoperative medication. The first study done by $\mathbf{N g}$, et al. (2006)and the second study done by Ulusoy, et al. (2016). Both studies found that tramadol did not cause cognitive impairment. These two studies are different from our study because they assessed cognitive effects of single therapeutic dose of tramadol, while our study assessed the cognitive effects of long-term supratherapeutic doses of tramadol.

Against our results, a study done by Zacny, et al. (2005)reported that neither tramadol $(50,100 \mathrm{mg}$ orally) nor morphine (25 mg orally) impaired psychomotor performance relative to placebo in sporadic drug users. But this study assessed the cognitive effects of low doses for short duration and the patients were recreational drug users.

Two studies revealed that acute tramadol administration (50-400 mg orally administered) did not impair performance in opioid-dependent volunteers (Carroll, et al. 2006; Lofwall, et al. 2007). Again, these two studies assessed the cognitive effects of low doses for short duration and the patients in both studies were opioid dependent and were suffering from opioid withdrawal manifestations.

The average dose of tramadol in the active group (Mean \pm SD) 1061.67 $\pm 781.29 \mathrm{mg}$ per day. The average dose of tramadol in the abstinent group (Mean \pm SD) 1147.5 $\pm \mathbf{5 5 8 . 5 2} \mathrm{mg}$ per day. The results in the current study found no effect of tramadol daily dose on cognitive performance. These results are in agreement with Bassiony, et al. (2017) who found no significant effect of the dose of tramadol on cognitive impairment. These results are consistent with the study of Mintzer, et al. (2010) who studied the effect of two doses of tramadol, $200 \mathrm{mg} /$ day and $800 \mathrm{mg} /$ day on cognitive performance and found no statistical difference between the two groups.

Duration of tramadol intake in the active group (Mean \pm SD) was 7.37 \pm 1.83 years. Duration of tramadol intake in the abstinent group (Mean \pm SD) was $\mathbf{6 . 5 3} \pm \mathbf{1 . 7 8}$ years. This study found no effect of increasing the duration of dependence on tramadol and deterioration in the cognitive performance. These results are in agreement with Bassiony, et al. (2017) who found no significant effect of the duration of tramadol use on cognitive impairment. These findings were also consistent with other works that found no significant correlation between the prolonged duration of administration of opioid analgesics and the worsening of cognitive performance (Sjogren, et al. 2000).

We did not find any significant correlation between nicotine use or seizures due to tramadol and cognitive impairment. These results were in agreement with Bassiony, et al. (2017) who found no significant effects of nicotine use or seizures due to tramadol on cognitive impairment. 
Effect of abstinence from tramadol on cognitive functions

In the abstinent group, we found statistically significant impairment in cognitive functions in the form of impaired total MoCAscores and impaired verbal fluency and delayed recall similar to active group. Thus, early abstinence from tramadol for 3 to 6 months did not reverse the cognitive impairments cause by tramadol addiction.

We did not find any study to assess the reversibility of cognitive impairment in tramadol addiction, but reversibility of cognitive impairments seen in different substances of abuse had been assessed in different studies.

When we reviewed the work done on reversibility of cognitive impairments in different substances of abuse, we found controversial data. Many neuropsychological studies of people with a history of substance use disorders have reported only minor cognitive deficits after long-term abstinence (Davies, et al. 2005, Davis, et al. 2002, Eckardt, et al. 1995, Fein, et al. 2006).

Some cross-sectional studies have not found a relationship between cognitive functioning and length of abstinence (Beatty, et al. 2000, Medina, et al. 2004), whereas there is strong evidence from longitudinal follow-up studies of people with substance use disorders that performance in many domains of cognitive functioning improves significantly during longterm abstinence(Bates, et al. 2005, Manning, et al. 2008, Pitel, et al. 2009, Rosenbloom, et al. 2007, Sullivan, et al. 2000, Zinn, et al. 2004). However, abstinence may have different effects on different domains of cognition, with memory functions possibly showing greater improvement than executive functioning or general cognitive ability (Bates, et al. 2005; Manning, et al. 2008; Rosenbloom, et al. 2007), and different profiles of improvement may be related to different substances (Di Sclafani, et al. 2002). Our explanation of the above results of different studies can be summarized as follows; first the duration of abstinence is different in different studies; second, the effects of different substances are different from each other; third, the various cognitive domains affected may be different in their rapidity of reversal after abstinence; fourth, affection of certain cognitive domains may preceed substance use; fifth, the type of therapy used during abstinence either pharmacotherapy or psychotherapy may have a role in improvement or persistence of certain cognitive deficits.

\section{LIMITATIONS}

This was a cross-sectional study and hence could not explain causation of the CI among patients with tramadol abuse. This clinical study included patients with drug-related problems with a high prevalence of comorbidities. Hence, these findings cannot be generalized to the general population. We included first degree relatives of the patients as control subjects. This might represent a potential bias in study design. We included consecutive patients without randomization, and this might lead to a lack of control for all confounders related to $\mathrm{CI}$.

\section{CONCLUSION}

Tramadol addiction causes cognitive impairment,mainly verbal fluency and memory impairment. Abstinence from tramadol for 3 to 6 months did not reverse these cognitive impairments. No effect of daily dose of tramadol on cognitive performance. No effect of duration of dependence on tramadol on cognitive performance.

\section{Recommendations}


We recommend future studies to: Include larger sample size, use of more detailed assessment tools for more accurate assessment of certain cognitive domains, assessment of cognitive functions after longer period of tramadol abstinence to determine if the cognitive impairments are reversible or not.

\section{REFERENCES}

1. Abo-Elmagd, S., Kodera, A., Okasha, T., Gawad, T.,and Rawson, R. (2013). Tramadol use in Egypt: The emergence of a new public health problem. Canadian Journal of Addiction Medicine, 4(1), p5.

2. Bassiony, M., Youssef, U., Hassan, M. S., Salah El-Deen, G. M., El-Gohari, H., Abdelghani, M., Abdalla, A. \& Ibrahim, D. H. (2017). Cognitive impairment and tramadol dependence. Journal of Clinical Psychopharmacology, 37(1),61-66.

3 . Bates, M. E., Voelbel, G. T., Buckman, J. F., Labouvie, E. W. \& Barry, D. (2005). Short-term neuropsychological recovery in clients with substance use disorders. Alcoholism, 29, 367-377.

4. Beatty, W. W., Tivis, R., Stott, H. D, Nixon, S. J. \& Parsons, O. A. (2000). Neuropsychological deficits in sober alcoholics: Influences of chronicity and recent alcohol consumption. Alcohol ClinExp Res, 24, 149-154.

5. Carroll, C. P., Walsh, S. L., Bigelow, G. E., Strain, E. C., \& Preston, K. L. (2006). Assessment of agonist and antagonist effects of tramadol in opioiddependent humans. ExpClinPsychopharmacol, 14(2), 109-120.

6. Davies, S. J. C., Pandit, S. A., Feeney, A., Stevenson, B. J., Kerwin, R. W., Nutt, D. J., Marshall, E. J., Boddington, S. \&Lingford-Hughes, A. (2005). Is there cognitive impairment in clinically 'healthy' abstinent alcohol dependence?. Alcohol Alcohol, 40, 498-503.

7. Davis, P. E., Liddiard, H. \& McMillan, T. M. (2002). Neuropsychological deficits and opiate abuse. Drug Alcohol Depend, 67, 105-108.

8. Di Sclafani, V., Ezekiel, F., Meyerhoff, D. J., et al. (1995). Brain atrophy and cognitive function in older abstinent alcoholic men. Alcohol ClinExp Res, 19, 1121-1126.

9. Eckardt, M. J., Stapleton, J. M., Rawlings, R. R., Davis, E. Z. \&Grodin, D. M. (1995). Neuropsychological functioning in detoxified alcoholics between 18 and 35 years of age. Am J Psychiatry, 152, 53-59.

10. Fein, G., Torres, J., Price, L. J. \& Di Sclafani, V. (2006). Cognitive performance in long-term abstinent alcoholic individuals. Alcohol ClinExp Res, 30, 1538-1544.

11 . Lofwall, M. R., Walsh, S. L., Bigelow, G. E., \& Strain, E. C. (2007). Modest opioid withdrawal suppression efficacy of oral tramadol in humans. Psychopharmacology, 194, 381-393.

12. Manning, V., Wanigaratne, S., Best, D., et al. (2008). Changes in neuropsychological functioning during alcohol detoxification. Eur Addict Res, 14, 226-233.

13. McLellan, A., et al. (1992). The fifth edition of the Addiction Severity Index. Journal of substance abuse treatment, 9(3), 199-213.

14. Medina, K. L., Shear, P. K., Schafer, J., Amstrong, T. G. \& Dyer, P. (2004). Cognitive functioning and length of abstinence in polysubstance dependent men. Arch ClinNeuropsychol, 19, 245258.

15. Mintzer, M. Z., Lanier, R. K., Lofwall, R. M., et al. (2010). Effects of repeated tramadol and morphine administration on psychomotor and cognitive performance in opioid-dependent volunteers. Drug Alcohol Depend, 111, 265-268.

16. Nasreddine, Z. S., Phillips, N. A., Bedirian, V., et al. (2005). The montrealcognitive assessment, MoCA: a brief screening tool for mild cognitiveimpairment. J Am GeriatrSoc, 53, 695-699.

17. Ng, K. F., Yuen, T. S., \& Ng, V. M. (2006). A comparison of postoperative cognitive function and pain relief with fentanyl or tramadol patient-controlled analgesia. J ClinAnesth, 18, 205-210.

18. Pitel, A. L., Rivier, J., Beaunieux, H., Vabret, F., Desgranges, B. \& Eustache, F. (2009). Changes in the episodic memory and executive functions of abstinent and relapsed alcoholics over a 6- 
SOHAG MEDICAL JOURNAL

Vol. 23 No.2 July 2018

month period. Alcohol ClinExp Res, 33, 490-498.

19 . Qassem, T., Beshry, Z., Assad, T., Omara, A., \&Abdmawgoud, M. (2003). Profiles of neurophysiological dysfunction in chronic heroin users. M.D degree thesis, faculty of medicine, Ain shams university.

20 . Rahman, T. T. and El Gaafary, M. M., (2009). Montreal Cognitive Assessment Arabic version: Reliability and validity prevalence of mild cognitive impairment among elderly attending geriatric clubs in Cairo. Geriatrics and Gerontology International, 9(1), 54-61.

21 . Reitan, R. (1958). Validity of the Trail Making Test as an indicator of organic brain damage. Perceptual and Motor Skills, 8, 271-276.

22. Rosenbloom, M. J., Rohlfing, T., O'Reilly, A. W., et al. (2007). Improvement in memory and static balance with abstinence in alcoholic men and women: Selective relations with change in brain structure. Psychiatry Res, 155, 91-102.

23. Shadnia, S., Soltaninejad, K., Heydari, K., Sasanian, G., \&Abdollahi, M. (2008). Tramadol intoxication: a review of 114 cases .Hum ExpToxicol, 27(3), 201205.

24. Sjogren, P., Thomsen, A. B., \& Olsen, A. K. (2000). Impaired neuropsychological performance in chronic nonmalignant pain patients receiving long-term oral opioid therapy. $J$ Pain Symptom Manage, 19(2), 100-108.

- 25. Sullivan, E. V., Rosenbloom, M. J., Lim, K. O. \&Pfefferbaum, A. (2000). Longitudinal changes in
Cognitive Profile in Tramadol Addicts Saber Hadad Mahdi

cognition, gait, and balance in abstinent and relapsed alcoholic men: Relationships to changes in brain structure. Neuropsychology, 14,178188.

26. Taghaddosinejad, F., Mehrpour, O., Afshari, R., Seghatoleslami, A., Abdollahi, M. (2011). Factors Related to Seizure in Tramadol Poisoning and Its Blood Concentration J Med Toxicol, 7(3), 183-188.

27. Ulusoy, H., Coskun, I., \&Arslan, M. (2016). Effects of midazolam or tramadol premedication on early cognitive function in endoscopic retrograde cholangiopancreatography (ERCP): A randomized, controlled, double-blind study. Journal of International Medical Research, 44(3), 542-556.

28. Zacny, J. P. (2005). Profiling the subjective, psychomotor, and physiological effects of tramadol in recreational drug users. Drug Alcohol Depend, 80, 273-278.

29. Zakaryaee, H., Mollazadeh, J., Aflakseir, A., Khormaei, F., \&Soofi, A. (2012). Cognitive Impairment in Methamphetamine, Buprenorphine and Tramadol Users. European Journal of Scientific Research, 68(3), 321-327.

30. Zinn, S., Stein, R. \&Swartzwelder, H. S. (2004). Executive functioning early in abstinence from alcohol. Alcohol ClinExp Res, 28, 1338-1346. 